\title{
The Apolar Locus of Two Tetrads of Points.
}

\author{
By Dr William P. Milure.
}

(Receired 30th November 1916. Read 12th January 1917).

1. In the present paper I propose to investigate the fundamental geometrical properties of the Apolar Locus of two tetrads of points in a plane.

The Apolar Locus of two tetrads of points $K, L, M, N$ and $P, Q, R, S$ is defined in the locus of the point $X$, moving so that the pencils $X[K, L, M, N]$ and $X[P, Q, R, S]$ are apolar.

In particular, $I$ wish to find in a convenient form the necessary and sufficient conditions which a quartic curve has to satisfy in order that it may be the Apolar Locus of two assigned tetrads.

2. We shall begin by proving some geometrical properties of a tetrad of points on a quartic curve, as these results will be required in the sequel.

\section{Lamra I.-The Principal Conics.}

Let $P, Q, R, S$ be a tetrad of points on the quartic curve

$$
F \equiv a x^{4}+b y^{4}+c z^{4}+6 f y^{2} z^{2}+6 g z^{2} x^{2}+6 h x^{2} y^{2}+\text { etc. }=0,
$$

no two of the four points being coincident.

Let the tetrad be regarded as the common points of the pencil of conics

$$
S_{1}+\lambda S_{2}=0
$$

Then it is plain that a unique pair of conics

$$
p S_{1}^{2}+2 q S_{1} S_{2}+r S_{2}^{2}=0
$$

of the system (2) can be so determined that the quartic

$$
F+p S_{1}^{2}+2 q S_{1} S_{2}+r S_{3}^{2}=0
$$

shall pass through the vertices of the self-polar triangle of the pencil $S_{1}+\lambda S_{2}$. For if we refer to the self-polar triangle as the triangle of reference and take the equations of $S_{1}$ and $S_{2}$ in the form

$$
\begin{aligned}
& S_{1} \equiv \alpha x^{2}+\beta y^{2}+\gamma z^{2} \\
& S_{2} \equiv x^{2}+y^{2}+z^{3},
\end{aligned}
$$


the conditions that the quartic (4) pass through the vertices of the triangle of reference are

$$
\begin{aligned}
& a+p \alpha^{2}+2 q \alpha+r=0 \\
& b+p \beta^{2}+2 q \beta+r=0 \\
& c+p \gamma^{2}+2 q \gamma+r=0
\end{aligned}
$$

which gives us unique values for $p, q, r$. The quartic we require is therefore (by (4) and (6))

$$
\left|\begin{array}{cccc}
F & S_{1}{ }^{2} & S_{1} S_{2} & S_{2}{ }^{2} \\
a & \alpha^{2} & \alpha & 1 \\
b & \beta^{2} & \beta & 1 \\
c & \gamma^{2} & \gamma & 1
\end{array}\right|=0 .
$$

We shall call (7) the "Auxiliary Quartic" of $F$ with respect to the tetrad $P, Q, R, S$. This Auxiliary Quartic possesses the property of passing through the four points of the tetrad and also the vertices of their self-polar triangle.

Furthermore, we shall call the conics obtained from (3) and (6), viz.

$$
\left|\begin{array}{cccc}
0 & S_{1}{ }^{2} & S_{1} S_{2} & S_{2}{ }^{2} \\
a & \alpha^{2} & \alpha & 1 \\
b & \beta^{2} & \beta & 1 \\
c & \gamma^{2} & \gamma & 1
\end{array}\right|=0
$$

the "Principal Conics" of the tetrad PQRS with respect to the quartic $F$.

\section{Lamma II.-The Generating Conic.}

If $S_{1}+\lambda S_{2}=0$ and $U_{1}+\mu U_{2}=0$ are two pencils of conics, and there is some member $S_{0}$ of the first pencil apolar (regarded as an envelope) to all members of the second, then the locus of the intersections of a member of the first (regarded as an envelope) with the member of the second (regarded as a locus) to which it is apolar, is a quartic through the base-points of the first pencil and the vertices of their common self-polar triangle.

For, if we state the condition that $S_{1}+\lambda S_{2}$ (regarded as an envelope) is apolar to $U_{1}+\mu U_{2}$ (regarded as a locus), we obtain a $(2,1)$ currespondence between $\lambda$ and $\mu$.

But, given $\mu$, one of the corresponding values of $\lambda$ thus obtained is the parameter of $S_{0}$. Hence, omitting this constant value in 
all cases, we obtain a $(1,1)$ correspondence between $\lambda$ and $\mu$. The required locus of the intersections of $S_{1}+\lambda S_{2}$ and $U_{1}+\mu U_{2}$ is therefore a quartic curve. To show that this quartic curve passes through the four points common to all members of the pencil $S_{1}+\lambda S_{2}$, we take one of these points $P$ and consider the member $U_{1}+\mu U_{2}$ which passes through $P$. A unique member of the pencil $S_{1}+\lambda S_{2}$ can be found apolar to this last-mentioned conic, and as $P$ is a point of intersection of both these corresponding conics, $P$ must lie on the quartic generated by such corresponding conics. Furthermore, the quartic passes through the vertices of the selfpolar triangle. For, if we choose $\lambda$ so that $S_{1}+\lambda S_{2}$ is a pair of straight lines, then the reciprocal of $S_{1}+\lambda S_{2}$ will be the tangential equation to the corresponding vertex $A$ of the self-polar triangle taken twice over. Hence the corresponding conic $U_{1}+\mu U_{2}$ being apolar (as a locus) to $A^{2}$ (as an envelope) passes through $A$; the result of which is that $A$ lies on a pair of corresponding conics, and consequently on the quartic. We call $S_{0}$ the Generating Conic of the quartic passing through the four given points and their self-polar triangle.

Conversely, any quartic through four points and their selfpolar triangle can be generated by the above method, and has a unique Generating Conic. For, any quartic through the points common to $S_{1}$ and $S_{2}$ can be expressed in the form $S_{1} T_{2}=S_{2} T_{1}$, which can be written in the more extended form

$$
\begin{aligned}
S_{1}\left(a_{2} x^{2}\right. & \left.+b_{2} y^{2}+c_{2} z^{2}+2 f_{2} y z+2 g_{2} z x+2 h_{2} x y\right) \\
& =S_{2}\left(a_{1} x^{2}+b_{1} y^{2}+c_{1} z^{2}+2 f_{1} y z+2 g_{1} z x+2 h_{1} x y\right) .
\end{aligned}
$$

Hence, using (5), the quartic (9) passes through the vertices of the self-polar triangle if

$$
\begin{aligned}
& \alpha a_{2}=a_{1} \\
& \beta b_{2}=b_{1} \\
& \gamma c_{2}=c_{1} .
\end{aligned}
$$

Now (9) can be written in the form

$$
S_{1}\left(T_{2}+\kappa S_{2}\right)=S_{2}\left(T_{1}+\kappa S_{1}\right)
$$

in which $S_{1}$ (envelope) is apolar to $T_{1}+\kappa S_{1}$ (locus), if

$$
\frac{a_{1}+\kappa \alpha}{\alpha}+\frac{b_{1}+\kappa \beta}{\beta}+\frac{c_{1}+\kappa \gamma}{\gamma}=0
$$


and also $S_{2}$ (envelope) is apolar to $T_{2}+\kappa S_{2}$ (locus), if

$$
\left(a_{2}+\kappa\right)+\left(b_{2}+\kappa\right)+\left(c_{2}+\kappa\right)=0 .
$$

Now (12) and (13) are consistent in virtue of $(10)$, which proves that every quartic through the common points of $S_{1}, S_{2}$ and the vertices of the self-polar triangle can be reduced to the form

$$
S_{1} U_{2}=S_{2} U_{1} \text {, }
$$

in which $S_{1}$ and $S_{3}$ (as envelopes) are respectively apolar to $U_{1}$ and $U_{2}$ (as loci).

We have still to show that $S_{1}+\lambda S_{2}$ (envelope) is apolar to its corresponding member of the $U$-pencil, viz. $U_{1}+\lambda U_{2}$.

Let us take

$$
\begin{aligned}
& S_{1} \equiv \alpha x^{2}+\beta y^{2}+\gamma z^{2} \\
& S_{2} \equiv x^{2}+y^{2}+z^{2} \\
& U_{1} \equiv a_{1} x^{2}+b_{1} y^{2}+c_{1} z^{2}+2 f_{1} y z+2 g_{1} z x+2 h_{1} x y \\
& U_{2} \equiv a_{2} x^{2}+b_{2} y^{2}+c_{2} z^{2}+2 f_{2} y z+2 g_{2} z x+2 h_{2} x y
\end{aligned}
$$

with the conditions

and

$$
\begin{aligned}
& \alpha a_{2}=a_{1} \\
& \beta b_{2}=b_{1} \\
& \gamma c_{2}=c_{1} \\
& \}
\end{aligned}
$$

$$
\left.\begin{array}{l}
\frac{a_{1}}{\alpha}+\frac{b_{1}}{\beta}+\frac{c_{1}}{\gamma}=0 \\
a_{2}+b_{2}+c_{3}=0
\end{array}\right\} \text {. }
$$

The two conditions (16) are equivalent in virtue of (15), and exist in virtue of the condition that $S_{1}$ and $S_{2}$ regarded as envelopes are to be apolar to $U_{1}$ and $U_{2}$ respectively, regarded as loci.

Now $S_{1}+\lambda S_{2}$ (envelope) will be apolar to $U_{1}+\lambda U_{2}$ (locus) if

$$
\frac{a_{1}+\lambda a_{2}}{\alpha+\lambda}+\frac{b_{1}+\lambda b_{2}}{\beta+\lambda}+\frac{c_{1}+\lambda c_{2}}{\gamma+\lambda}=0
$$

i.e. if, by using (15),

$$
\frac{a_{2}(\alpha+\lambda)}{\alpha+\lambda}+\frac{b_{2}(\beta+\lambda)}{\beta+\lambda}+\frac{c_{2}(\gamma+\lambda)}{\gamma+\lambda}=0
$$

which is true in virtue of (16).

We have thus shewn that every such Seven-Point Quartic passing through the four points of a quadrangle and the vertices 
of the self-polar triangle can be generated by the intersections of the corresponding members of two pencils of conics $S_{1}+\lambda S_{2}$ and $U_{1}+\lambda U_{2}$, in which every member of the $S$-pencil regarded as an envelope is apolar to every member of the $U$-pencil regarded as a locus.

It still remains to demonstrate the existence of the unique "generating-conic." Using the notation of (14), we have to show that a unique member of the $S$-pencil exists, which, regarded as an envelope, is apolar to every member of the $U$-pencil regarded as a locus. Let the required generating-conic be $S_{1}+\rho S_{2}$, and let it, if possible, be apolar to $U_{1}+\lambda U_{2}$ for all values of $\lambda$. This requires the condition

i.e. using (15),

$$
\frac{a_{1}+\lambda a_{2}}{\alpha+\rho}+\frac{b_{1}+\lambda b_{2}}{\beta+\rho}+\frac{c_{1}+\lambda c_{2}}{\gamma+\rho}=0
$$

which becomes

$$
\frac{a_{2}(\alpha+\lambda)}{\alpha+\rho}+\frac{b_{2}(\beta+\lambda)}{\beta+\rho}+\frac{c_{2}(\gamma+\lambda)}{\gamma+\rho}=0
$$

$$
(\rho-\lambda)\left\{\left(a_{2} \alpha+b_{2} \beta+c_{3} \gamma\right) \rho-\left(a_{2} \beta \gamma+b_{2} \gamma \alpha+c_{2} \alpha \beta\right)\right\}=0,
$$

after some reduction with the help of (15).

Hence, neglecting the factor $\rho-\lambda$, we obtain the required value for $\rho$, and the equation of the generating-conic becomes

$$
\left(a_{2} \alpha+b_{2} \beta+c_{2} \gamma\right) S_{1}+\left(a_{2} \beta \gamma+b_{2} \gamma \alpha+c_{2} \alpha \beta\right) S_{2}=0 .
$$

Hence we have the following result :-

Any quartic through four points and the vertices of their selfpolar triangle can be generated by the method just described. For every such quartic the method of generation is unique, and to it corresponds a single member of the pencil, its "generating conic."

3. We shall next require some of the geometrical properties existing between a pencil of conics $S_{1}+\lambda S_{2}$ and a Class-Quartic,

$$
\begin{gathered}
\psi_{l}{ }^{4} \equiv A l^{4}+B m^{4}+C n^{4}+6 F m^{2} n^{2}+6 G n^{2} l^{2}+6 H l^{2} m^{2}+\text { etc. } \\
\text { LemaA III.-The Self-Conjugate Conics. }
\end{gathered}
$$

Given any conic $S_{1}+\lambda S_{2}$ of the $S$-pencil, there exists a unique conic $S_{1}+\mu S_{2}$ belonging to the pencil, such that $\left(S_{1}+\lambda S_{2}\right)\left(S_{1}+\mu S_{2}\right)$ regarded as a quartic-locus is apolar to $\psi_{i}{ }^{4}$ regarded as a quartic envelope. 
For if

$$
\begin{aligned}
& S_{1} \equiv \alpha x^{2}+\beta y^{2}+\gamma z^{2} \\
& S_{2} \equiv x^{2}+y^{2}+z^{2}
\end{aligned},
$$

then $\left(S_{1}+\lambda S_{2}\right)\left(S_{1}+\mu S_{2}\right)$ is apolar to $\psi_{t}{ }^{4}=0$ if

$$
\begin{aligned}
A(\alpha+\lambda) & (\alpha+\mu)+B(\beta+\lambda)(\beta+\mu)+C(\gamma+\lambda)(\gamma+\mu) \\
& +F\{(\beta+\lambda)(\gamma+\mu)+(\beta+\mu)(\gamma+\lambda)\} \\
& +G\{(\gamma+\lambda)(\alpha+\mu)+(\gamma+\mu)(\alpha+\lambda)\} \\
& +H\{(\alpha+\lambda)(\beta+\mu)+(\alpha+\mu)(\beta+\lambda)\}=0 .
\end{aligned}
$$

This is a $(1,1)$ algebraic correspondence between $\lambda$ and $\mu$, having in general two self-corresponding members. We shall call these the two "self-conjugate" conics of the pencil.

\section{Lamma IV.-The Polo-Reciprocal Conic.}

If $l$ typify the tangential coordinates of a given line, then $\psi_{i}{ }^{2} \psi_{l}{ }^{2}=0$ is the polar conic of the line $l^{\prime}$ with respect to the classquartic $\psi_{l}{ }^{4}$. The conic-envelope $\psi_{2}{ }^{2} \psi_{l}{ }^{2}$ is apolar to the conic locus $a_{x}^{2}=0$ if $\psi_{u l}{ }^{2} \psi_{a}^{2}=0$. Hence $\psi_{l}^{2} \psi_{a}^{2}$ is the locus of lines whose polar conics are apolar to $a_{x}{ }^{2}$, in virtue of which property $\psi_{z}{ }^{2} \psi_{a}{ }^{2}$ is usually called the polar conic of $a_{x}^{2}$ with respect to $\psi_{2}^{4}$. Now the polar conics of all the members of the pencil $S_{1}+\lambda S_{2}$ of (19) plainly form a pencil of conic envelopes

Let

$$
\Pi_{1}+\lambda \Pi_{2}=0 \text {. }
$$

$$
\Pi_{1} \equiv A_{1} l^{2}+B_{1} m^{2}+C_{1} n^{2}+2 F_{1} m n+2 G_{1} n l+2 H_{1} l m=0,
$$

and $\Pi_{2} \equiv A_{2} l^{2}+B_{2} m^{2}+C_{2} n^{2}+2 F_{2} m n+2 G_{2} n l+2 H_{2} l m=0$.

It has to be noted that the conics $S_{1} \equiv a_{x}^{2}$ and $S_{2} \equiv a_{x}^{\prime 2}$ are together apolar to $\psi_{l}{ }^{4}=0$ if $\psi_{a}{ }^{2} \psi_{a^{\prime}}{ }^{2}=0$, i.e. if $S_{1}$ be apolar to $\Pi_{2}$ or $S_{2}$ apolar to $\Pi_{1}$ (these being equivalent conditions, each requiring the vanishing of $\left.\psi_{a}^{2} \psi_{a^{2}}{ }^{2}\right)$.

Hence, if $I_{12}$ denote the invariant expressing the condition that $S_{1} S_{2}$ be apolar to $\psi_{2}^{4}$, we have

$$
\begin{aligned}
& I_{11} \equiv \alpha A_{1}+\beta B_{1}+\gamma C_{1} \ldots \ldots \ldots \ldots \ldots . \\
& I_{12} \equiv \alpha A_{2}+\beta B_{2}+\gamma C_{2} \equiv A_{2}+B_{2}+C_{2} \\
& I_{22} \equiv A_{2}+B_{2}+C_{2} .
\end{aligned}
$$

It will also be convenient to use the following abbreviation :-

$$
\begin{aligned}
& P \equiv \alpha^{2} A_{2}+\beta^{2} B_{2}+\gamma^{2} C_{2} \\
& Q \equiv \beta \gamma A_{1}+\gamma \alpha B_{1}+\alpha \beta C_{1} .
\end{aligned}
$$


We therefore have, on expressing the condition that $S_{1}+\lambda S_{2}$ be apolar to $\Pi_{1}+\lambda \Pi_{2}$, the equation

$$
\lambda^{2} I_{22}+2 \lambda I_{12}+I_{11}=0,
$$

giving the parameters of the two "self-conjugate conics" with respect to $\psi_{2}{ }^{4}$ of the $S_{1}, S_{2}$ pencil.

Let us now form the reciprocal of the conic $\Pi_{1}+\mu \Pi_{2}$ with respect to the conic $S_{1}+\lambda S_{2}$. We obtain

$$
\begin{array}{r}
\left(A_{1}+\mu A_{2}\right)(\alpha+\lambda)^{2} x^{2}+\left(B_{1}+\mu B_{2}\right)(\beta+\lambda)^{2} y^{2}+\left(C_{1}+\mu C_{2}\right)(\gamma+\lambda)^{2} z^{2} \\
+ \text { etc }=0 .
\end{array}
$$

The conic (28) will be apolar to the conic $S_{1}+\mu S_{2}$ (regarded as an envelope) if

that is, if

$$
\Sigma(\beta+\mu)(\gamma+\mu)(\alpha+\lambda)^{2}\left(A_{1}+\mu A_{2}\right)=0,
$$

$$
\begin{aligned}
(\mu+\alpha)(\mu+\beta)(\mu & +\gamma)\left(I_{22} \lambda^{2}+2 I_{12} \lambda+I_{11}\right) \\
& +(\lambda-\mu)^{2}\left(\mu \overline{P-I_{11}}+\overline{Q-\alpha \beta \gamma I_{22}}\right)=0 .
\end{aligned}
$$

Hence, between $\lambda$ and $\mu$ exists a $(2,3)$ correspondence defined by the above relationship.

Putting $I_{22} \lambda^{2}+2 I_{12} \lambda+I_{11}$ equal to zero, we see that to either of the self-conjugate conics correspond themselves (taken twice over) and another conic whose parameter is given by

$$
\left(P-I_{11}\right) \mu+\left(Q-\alpha \beta \gamma I_{22}\right)=0 \text {. }
$$

Conversely, to the conic (30) correspond the self-conjugate conics.

The conic (30) is thus uniquely defined, and we shall call it the "Polo-Reciprocal Conic" of the $S_{1}, S_{2}$ pencil.

\section{The Apolar Locus.}

If $\phi_{l}^{4}=0$ and $\psi_{l}^{4}=0$ be the equations of two class-quartics, the equation to the locus of a point $X$, moving so that the four tangents from $X$ to $\phi_{l}{ }^{4}$ apolarly separate those from $X$ to $\psi_{l}{ }^{4}$, is

$$
(\phi \psi x)^{4}=0 \text {. }
$$

We shall call the quartic (31) the "Apolar Locus" of $\phi_{l}^{4}$ and $\psi_{i}^{4}$.

If, again, $\phi_{l}{ }^{4}$ consist of four points, we may regard these points as the intersection of a pencil of conics, viz.,

$$
\begin{aligned}
& S_{1} \equiv a_{z}{ }^{2} \equiv b_{x}{ }^{2}=0 \\
& S_{z} \equiv a_{z}{ }^{2} \equiv b_{x}{ }^{2}=0 .
\end{aligned}
$$


In this case

$$
\phi_{l}{ }^{4} \equiv\left(a a^{\prime} l\right)^{2}\left(b b^{\prime} l\right)^{2}-(a b l)^{2}\left(a^{\prime} b^{\prime} l\right)^{2}=0 .
$$

Hence, by (31) and (33) the apolar locus is

$$
\left(a a^{\prime} \widehat{\psi x}\right)^{2}\left(b b^{\prime} \widehat{\psi x}\right)^{2}-(a b \widehat{\psi x})^{2}\left\langle a^{\prime} b^{\prime} \widehat{\psi x}\right)^{2}=0,
$$

where $\widehat{\psi x}$ typifies $\left(\psi_{2} x_{3}-\psi_{3} x_{2}\right)$ in the usual way.

On multiplying out and reducing (34), we obtain

$$
\begin{aligned}
& \phi_{a}{ }^{2} \phi_{b}{ }^{2} S_{2}{ }^{2}-2 \phi_{a}{ }^{2} \phi_{a^{\prime}}{ }^{2} S_{1} S_{2}+\phi_{a^{\prime}}{ }^{2} \phi_{b^{\prime}}{ }^{2} S_{1}{ }^{2} \\
& =4 S_{1}\left(\phi_{b}{ }^{2} \phi_{a} \phi_{a} a_{x} a_{x}^{\prime}-\phi_{b}{ }^{2} \phi_{a} \phi_{b} a_{x}^{\prime} b_{x}{ }^{\prime}\right) \\
& +4 S_{2}\left(\phi_{a}{ }^{2} \phi_{b} \phi_{b}{ }^{\prime} b_{x} b_{x}{ }^{\prime}-\phi_{b^{\prime}}{ }^{2} \phi_{a} \phi_{b} a_{x} b_{x}\right) .
\end{aligned}
$$

In accordance with the notation of Lemma IV., the equation (35) may be written

$$
\begin{aligned}
& \left(I_{11} S_{2}^{2}-2 I_{12} S_{1} S_{2}+I_{22} S_{1}^{2}\right) \\
& =S_{1}\left\{\begin{array}{l}
\left(A_{2} \alpha x^{2}+B_{2} \beta y^{2}+C_{2} \gamma z^{2}+F_{2} \overline{\beta+\gamma} y z+G_{2} \overline{\gamma+\alpha} z x+H_{2} \overline{\alpha+\beta} x y\right) \\
-\left(A_{1} x^{2}+B_{1} y^{2}+C_{1} z^{2}+2 F_{1} y z+2 G_{1} z x+2 H_{1} x y\right)
\end{array}\right\} \\
& +S_{2}\left\{\begin{array}{l}
\left(A_{1} \alpha x^{2}+B_{1} \beta y^{2}+C_{1} \gamma z^{2}+F_{1} \overline{\beta+\gamma} y z+G_{1} \overline{\gamma+\alpha} z x+H_{1} \overline{\alpha+\beta} x y\right) \\
-\left(A_{2} \alpha^{2} x^{2}+B_{2} \beta^{2} y^{2}+C_{2} \gamma^{2} z^{2}+2 F_{2} \beta \gamma y z+2 G_{2} \gamma \alpha z x+2 H_{2} \alpha \beta x y\right.
\end{array}\right\}
\end{aligned}
$$

or in the still shorter form

$$
\left(I_{11} S_{2}^{2}-2 I_{12} S_{1} S_{2}+I_{22} S_{1}^{2}\right)=S_{1} V_{2}+S_{2} V_{1} .
$$

It is plain that the right-hand side of (36) represents a quartic passing through the four points of the $S_{1}, S_{2}$ pencil, and also through the vertices of their self-polar triangle, inasmuch as the terms $x^{4}, y^{4}, z^{4}$ are absent. Furthermore, the left-hand side represents the combined equation to two conics of the $S_{1}, S_{2}$ pencil. We therefore have the following results :-

The Principal Conics of the Apolar Locus with respect to the generating tetrad of points $\phi_{l}{ }^{4}$ are the Self-Conjugate Conics of the $S_{1}, S_{2}$ pencil with respect to $\psi_{l}^{4}$; and vice-versa with respect to $\psi_{i}{ }^{4}$ and $\phi_{l}^{4}$.

The right-hand side of (36) is the Auxiliary Quartic of the Apolar Locus with respect to the $\phi$-tetrad.

To find the Generating Conic of the above Auxiliary Quartic, we note that its equation in (36) or (37) is in normal form, inasmuch as $S_{1}$ (envelope) is apolar to the conic $V_{1}$ (locus) and 
$S_{2}$ to $\nabla_{2}$, and we have therefore only to express the condition that $S_{1}+\mu S_{2}$ is apolar to every conic of the $V_{1}+\lambda V_{3}$ pencil.

The condition required is

$$
\Sigma(\beta+\mu)(\gamma+\mu)\left\{\left(A_{1} \alpha-A_{2} \alpha^{2}\right)+\lambda\left(A_{2} \alpha-A_{1}\right)\right\}=0,
$$

i.e. $\quad \Sigma(\beta+\mu)(\gamma+\mu)(\alpha-\lambda)\left(A_{1}-A_{2} \alpha\right)=0$,

i.e. $\quad(\lambda+\mu)\left\{\mu\left(P-I_{11}\right)+\left(Q-\alpha \beta \gamma I_{22}\right)\right\}=0$.

Neglecting the irrelevant factor $(\lambda+\mu)$ and comparing (39) with (30), we obtain that the Generating Conic of the Apolar Locus relative to the $\phi$-tetrad is the Polo-Reciprocal Conic of $S_{1}, S_{2}$ pencil relative to $\psi_{2}{ }^{4}$.

Hence the generating conic of the Apolar Locus with respect to either generating tetrad is the Polo-Reciprocal Conic of that tetrad with respect to the other generating tetrad.

5. We are now in a position to attack the fundamental problem of this paper, viz., the necessary and sufficient conditions that a quartic must satisfy, in order that it may be the Apolar Locus of two assigned tetrads.

We have to show that if a quartic curve pass through two tetrads of points (which we shall call the $\phi$-and the $\psi$-tetrads respectively), and is such that :-

(1) the $\phi$-Principal Conics are the Self-Conjugate Conics of $\phi$ with respect to $\psi_{i}^{4}$;

(2) the $\psi$-Principal-Conics are the Self-Conjugate Conics of $\psi$ with respect to $\phi_{i}{ }^{4}$;

(3) the $\phi$-Generating-Conic is the Polo-Reciprocal Conic of $\phi$ with respect to $\psi_{i}^{*}$;

(4) the $\psi$-Generating Conic is the Polo-Reciprocal Conic of $\psi$ with respect to $\phi_{l}{ }^{4}$;

then, subject to these conditions, the quartic curve is the Apolar Locus of the $\phi$ - and $\psi$-tetrads.

We have already seen under Lemmas I. and II. that if the most general form of quartic passing through the four intersections of

$$
\begin{aligned}
& S_{1} \equiv \alpha x^{2}+\beta y^{2}+\gamma z^{2}=0 \\
& S_{2} \equiv x^{2}+y^{2}+z^{2}=0
\end{aligned}
$$


(i.e. the $\phi_{l}{ }^{*}$-tetrad) and possessing given Principal Conics, viz. $I_{11} S_{2}^{2}-2 I_{12} S_{1} S_{2}+I_{22} S_{1}^{3}=0$ (the notation being that of (22), (23), (24), $)$ is

$$
\begin{aligned}
& \rho\left(I_{11} S_{2}^{2}-2 I_{12} S_{1} S_{2}+I_{22} S_{1}^{2}\right) \\
& \quad=S_{1}\left(a_{2} x^{2}+b_{2} y^{2}+c_{2} z^{2}+2 f_{2} y z+2 g_{2} z x+2 h_{2} x y\right) \\
& \quad-S_{2}\left(\alpha a_{2} x^{2}+\beta b_{2} y^{2}+\gamma c_{2} z^{2}+2 f_{1} y z+2 g_{1} z x+2 h_{1} x y\right),
\end{aligned}
$$

where the right-hand side is the Auxiliary Quartic expressed in normal form according to $(10)$, if

$$
a_{2}+b_{2}+c_{2}=0 \text {. }
$$

The generating conic of the right-hand side is obtained by expressing the condition that $S_{1}+\mu S_{2}$ is apolar to every conic of the system

$$
\begin{aligned}
\left(\alpha a_{2} x^{2}\right. & \left.+\beta b_{2} y^{2}+\gamma c_{2} z^{2}+2 f_{1} y z+2 g_{1} z x+2 h_{1} x y\right) \\
& +\lambda\left(a_{2} x^{2}+b_{2} y^{2}+c_{2} z^{2}+2 f_{2} y z+2 g_{2} z x+2 h_{2} x y\right)=0 .
\end{aligned}
$$

The condition required is

$$
\Sigma(\beta+\mu)(\gamma+\mu)(\alpha+\lambda) a_{2}=0,
$$

i.e. $\quad(\lambda-\mu)\left\{\mu\left(\alpha a_{2}+\beta b_{2}+\gamma c_{2}\right)-\left(\beta \gamma a_{2}+\gamma \alpha b_{3}+\alpha \beta c_{2}\right)\right\}=0$.

Casting aside the irrelevant factor $\lambda-\mu$, we obtain that the generating-conic is

$$
\left(\alpha a_{2}+\beta b_{2}+\gamma c_{2}\right) S_{1}+\left(\beta \gamma a_{2}+\gamma \alpha b_{2}+\alpha \beta c_{2}\right) S_{2}=0 .
$$

Now the generating-conic is to be (39), namely,

$$
\left(P-I_{11}\right) S_{1}+\left(\alpha \beta \gamma I_{22}-Q\right) S_{2}=0,
$$

i.e. $\quad\left\{\alpha\left(\alpha A_{2}-A_{1}\right)+\beta\left(\beta B_{2}-B_{1}\right)+\gamma\left(\gamma C_{2}-C_{1}\right)\right\} S_{1}$

$$
+\left\{\beta \gamma\left(\alpha A_{2}-A_{1}\right)+\gamma \alpha\left(\beta B_{2}-B_{1}\right)+\alpha \beta\left(\gamma C_{2}-C_{1}\right)\right\} S_{2}=0 \text {. }
$$

Identifying (43) with (44), and using (42), we find that

$$
\begin{aligned}
& a_{2}=-\sigma\left(\alpha A_{2}-A_{1}\right) \\
& b_{2}=-\sigma\left(\beta B_{2}-B_{1}\right) \\
& c_{2}=-\sigma\left(\gamma C_{2}-C_{1}\right),
\end{aligned}
$$

The most general quartic, therefore, (1) which passes through the $\phi$-tetrad, (2) which has as its $\phi$-Principal-Conics the Self-Conjugate 
Conics of $\phi$ with respect to $\psi_{l}^{4},(3)$ which has as its $\phi$-GeneratingConic the Polo-Reciprocal of $\phi$ with respect to $\psi_{l}^{4}$, is

$$
\begin{aligned}
& \rho\left(I_{11} S_{2}^{2}-2 I_{12} S_{1} S_{2}+I_{22} S_{1}^{2}\right) \\
& +\sigma\left[\left\{\left(\alpha A_{2}-A_{1}\right) x^{2}+\left(\beta B_{2}-B_{1}\right) y^{2}+\left(\gamma C_{2}-C_{1}\right) z^{2}\right\} S_{1}\right. \\
& \left.\quad+\left\{\alpha\left(A_{1}-\alpha A_{2}\right) x^{2}+\beta\left(B_{1}-\beta B_{2}\right) y^{2}+\gamma\left(C_{1}-\gamma C_{2}\right) z^{2}\right\}\right] \\
& +S_{1}\left(p_{2} y z+q_{2} z x+r_{2} x y\right)+S_{2}\left(p_{1} y z+q_{1} z x+r_{1} x y\right)=0,
\end{aligned}
$$

where $\rho, \sigma, p_{1}, q_{1}, r_{1}, p_{2}, q_{2}, r_{2}$ are arbitrary constants.

We thus see that for a quartic to pass through four specified points, and have thereat the given conics as Principal and Generating Conics respectively, is tantamount to seven linear conditions. Now, if we make this quartic satisfy the corresponding conditions with regard to the $\psi$-tetrad of points, we shall obtain other seven linear conditions, i.e. seven linear equations connecting $\rho, \sigma, p_{1}, q_{1}, r_{1}, p_{2}, q_{2}, r_{2}$. Let these linear conditions be typified by

$$
\begin{array}{r}
R_{\kappa}{ }^{\prime \prime} \rho+S_{\kappa} \sigma+P_{\kappa} p_{1}+Q_{\kappa} q_{1}+R_{\kappa} r_{1}+P_{\kappa}{ }^{\prime} p_{2}+Q_{\kappa}{ }^{\prime} q_{2}+R_{\kappa}{ }^{\prime} r_{2}=0 \\
(\kappa=1,2,3,4,5,6,7) .
\end{array}
$$

These equations will yield a unique solution for

$$
\rho: \sigma: p_{1}: q_{1}: r_{1}: p_{2}: q_{2}: r_{2}
$$

unless the determinants of the matrix

$$
\left\|R_{\kappa}^{\prime \prime}, S_{\kappa}, P_{\kappa}, Q_{\kappa^{\prime}}, R_{\kappa}, P_{\kappa}^{\prime}, Q_{\kappa}^{\prime}, R_{\kappa}^{\prime}\right\|
$$

severally vanish. To show that they do not vanish in general, it will be sufficient to show that they do not vanish for a particular case. I have worked out in detail the test case of $\psi_{l}^{4}$ the intersections of the two conics

$$
\begin{aligned}
& 3 x^{2}-2 y^{2}+5 z^{2}+6 y z+14 z x-8 x y=0 \\
& 7 x^{2}-11 y^{2}+z^{2}-4 y z-10 z x+12 x y=0
\end{aligned}
$$

and $\phi_{l}{ }^{4}$ the four points $(1, \pm 1, \pm 1)$. The details are too long for reproduction here. Hence in general we get a unique solution for $\rho: \sigma: p_{\mathrm{r}}: q_{1}: r_{1}: p_{2}: q_{2}: r_{2}$, and as these are known to be satisfied by the coefficients of the Apolar Locus of $\phi_{l}{ }^{4}$ and $\psi_{l}{ }^{4}$, the Apolar Locus must be the unique solution required. 
6. The Clebschian Quartic with a degenerate Apolar Conic.

The Clebschian Quartic with a degenerate Apolar Conic is particularly interesting from the point of view of one particular method of its apolar generation.

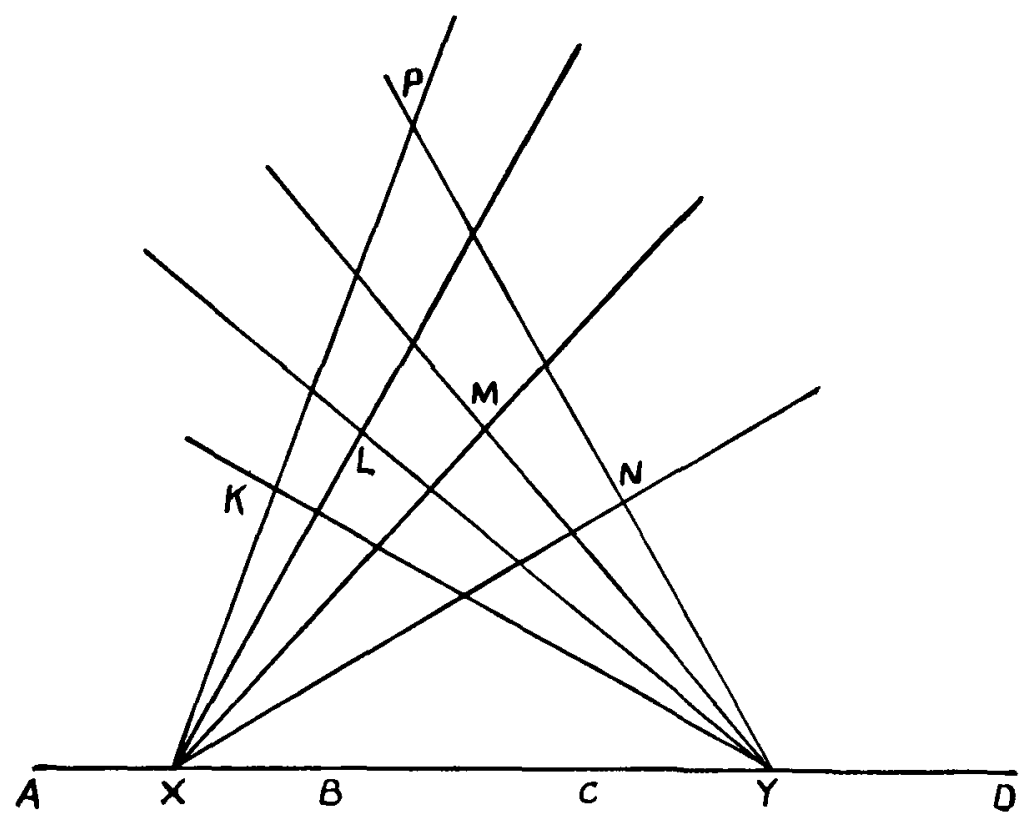

Let $X, Y$ be the degenerate Apolar Conic of the given Clebschian Quartic. Let the curve cut the line $X Y$ in the points $A B C D$. We know that the range $[A B C D]$ is equianharmonic, and has $X^{2}, Y^{2}$ as its Hessian Points. Now consider the four points $K, L, M, N$, in which the first, second, third, and fourth lines of the $X$-pencil eut the first, second, third, and fourth lines of the $Y$-pencil respectively. Consider the Apolar Locus of $A, B, C, D$ and $K, L, M, N$, which, of course, passes through these eight points. Now $[A, B, C, D]$, being equianharmonic, is apolar to $X, Y$ and any other two collinear points. Hence $P[A B C D]$ is apolar to $P[K, L, M, N]$. Consequently, $P$ lies on the Apolar Locus. We thus see that the Apolar Locus passes through the 
sixteen intersections of the $X$-pencil with the $Y$ pencil, and is, in fact, the Clebschian Quartic, having $X, Y$ as its degenerate Apolar Conic.

Conversely, if we wish to apolarly generate a given Clebschian Quartic having $X, Y$ as its Apolar Conic, we draw any line $x_{1}$ through $X$, join the four intersections of $x_{1}$ with the curve to $Y$, obtaining $y_{1}, y_{2}, y_{3}, y_{4}$. By joining up the remaining intersections of $y_{1}, y_{2}, y_{3}, y_{4}$ with the curve to $X$, we obtain $x_{2}, x_{3}, x_{4}$. Then the tetrads of points given by the following scheme will, along with $A B C D$, apolarly generate the given Clebschian Quartic.

\begin{tabular}{|c|c|c|c|}
\hline$x_{1}$ & $x_{2}$ & $x_{3}$ & $x_{4}$ \\
\hline \hline$y_{1}$ & $y_{2}$ & $y_{3}$ & $y_{4}$ \\
\hline$y_{2}$ & $y_{1}$ & $y_{4}$ & $y_{3}$ \\
\hline$y_{3}$ & $y_{4}$ & $y_{1}$ & $y_{2}$ \\
\hline$y_{4}$ & $y_{3}$ & $y_{2}$ & $y_{1}$ \\
\hline
\end{tabular}

The first two rows taken together give the points $x_{1} y_{1}, x_{2} y_{2}$, $x_{3} y_{3}, x_{4} y_{4}$, which is a tetrad co-apolar with $A B C D$.

Also, the first and third row taken together give the points $x_{1} y_{3}, x_{2} y_{1}, x_{3} y_{4}, x_{4} y_{3}$, which is another tetrad co-apolar with $A B C D$.

Again, reverting to the figure of this article, the tangents at $A B C D$ are concurrent; for, if $A T$ be the tangent at $A, A\left[D^{s} T\right]$ is apolar to $A[K L M N]$, i.e. $A T$ passes through the pole of $A D$ with respect to the tetrad $A B C D$.

Many properties of the Clebschian Quartic regarding the pencil of conics through $K L M N$ and all such tetrads now follow at once from our discussion of the general method of apolarly generating the general Quartic Curve. 


\section{Two Tetrads on the same Conic.}

The case when the $\phi$ - and the $\psi$-tetrads both lie on the same conic yields a rather unexpected result. We find on investigation that, not a unique quartic, but a pencil of quartics satisfy the criteria of $\S 5$. I have, however, not completed the solution at the present date. 